OPEN ACCESS

Edited by:

Sonja A. Kotz,

Maastricht University, Netherlands

Reviewed by:

Mireille Besson,

Centre National de la Recherche

Scientifique, France

LouAnn Gerken,

University of Arizona, USA

*Correspondence:

Jia Hoong Ong

jia.ong@westernsydney.edu.au

Specialty section:

This article was submitted to

Language Sciences,

a section of the journal

Frontiers in Psychology

Received: 10 March 2016

Accepted: 27 July 2016

Published: 08 August 2016

Citation:

Ong JH, Burnham D, Stevens CJ

and Escudero P (2016) Naïve

Learners Show Cross-Domain

Transfer after Distributional Learning:

The Case of Lexical and Musical

Pitch. Front. Psychol. 7:1189.

doi: 10.3389/fpsyg.2016.01189

\section{Naïve Learners Show Cross-Domain Transfer after Distributional Learning: The Case of Lexical and Musical Pitch}

\author{
Jia Hoong Ong *, Denis Burnham, Catherine J. Stevens and Paola Escudero \\ The MARCS Institute for Brain, Behaviour and Development, Western Sydney University, Sydney, NSW, Australia
}

Experienced listeners of a particular acoustic cue in either speech or music appear to have an advantage when perceiving a similar cue in the other domain (i.e., they exhibit cross-domain transfer). One explanation for cross-domain transfer relates to the acquisition of the foundations of speech and music: if acquiring pitch-based elements in speech or music results in heightened attention to pitch in general, then cross-domain transfer of pitch may be observed, which may explain the cross-domain phenomenon seen among listeners of a tone language and listeners with musical training. Here, we investigate this possibility in naïve adult learners, who were trained to acquire pitchbased elements using a distributional learning paradigm, to provide a proof-of-concept for the explanation. Learners were exposed to a stimulus distribution spanning either a Thai lexical tone minimal pair or a novel musical chord minimal pair. Within each domain, the distribution highlights pitch to facilitate learning of two different sounds (Bimodal distribution) or the distribution minimizes pitch so that the input is inferred to be from a single sound (Unimodal distribution). Learning was assessed before and after exposure to the distribution using discrimination tasks with both Thai tone and musical chord minimal pairs. We hypothesize: (i) distributional learning for learners in both the tone and the chord distributions, that is, pre-to-post improvement in discrimination after exposure to the Bimodal but not the Unimodal distribution; and (ii) for both the tone and chord conditions, learners in the Bimodal conditions but not those in the Unimodal conditions will show cross-domain transfer, as indexed by improvement in discrimination of test items in the domain other than what they were trained on. The results support both hypotheses, suggesting that distributional learning is not only used to acquire the foundations of speech and music, but may also play a role in cross-domain transfer: as a result of learning primitives based on a particular cue, learners show heightened attention to that cue in any auditory signal.

Keywords: cross-domain transfer, distributional learning, statistical learning, language and music, pitch

\section{INTRODUCTION}

Language and music are two human universals that share many commonalities. For instance, both consist of discrete elements which are organized in a hierarchical structure governed by rules or syntax (Patel, 2003): in language, phonemes are organized into syllables, which are then organized into words and sentences; in music, notes are organized into chords, which then comprise musical 
phrases. Furthermore, spoken language (speech) and music rely on similar acoustic dimensions in their expression such as rhythm, pitch, intensity, and timbre-although the relative importance of each dimension depends on the language or music system in question (Patel, 2008).

Given the many commonalities between the two, the question arises whether having experience in one domain may be advantageous for perceiving and learning the other domain, that is, whether cross-domain transfer is possible. There is evidence of music-to-speech transfer in various aspects of language (Besson et al., 2011) such as syllabic perception (Musacchia et al., 2007; Ott et al., 2011; Elmer et al., 2012; Kühnis et al., 2013; Chobert et al., 2014; Bidelman and Alain, 2015), phonological ability (Slevc and Miyake, 2006; Herrera et al., 2011) and word segmentation (François et al., 2012). Furthermore, musicians show higher performance relative to non-musicians in identifying and discriminating lexical tones-a linguistic device used in tone languages in which meaning can be differentiated by a constellation of cues, primarily pitch (e.g., in Mandarin, $/ \mathrm{ma} 55 /{ }^{1}$ refers to 'mother' while /ma51/ refers to 'to scold'; Yip, 2002). The advantage musicians enjoy is seen both at the level of behavior (Alexander et al., 2005; Wong and Perrachione, 2007; Burnham et al., 2014) and electrophysiology (Wong et al., 2007; Marie et al., 2011). Since musicians generally have extensive training in perceiving, producing, and attending to musical pitch, it appears that they are able to transfer their expertise with musical pitch to perceive linguistic pitch.

Transfer also occurs in the other direction, that is, extensive experience with a particular speech cue can be transferred to other non-speech/music domains. For example, native Finnish listeners, whose language is a quantity language (i.e., it distinguishes words based on vowel duration), are more accurate at detecting durational deviants of non-speech sounds than native French listeners, whose language does not use duration to distinguish meaning (Marie et al., 2012). In terms of musical pitch, tone language listeners are more successful than non-tone language listeners in discriminating and producing sung intervals (Pfordresher and Brown, 2009); in tracking pitch of non-speech stimuli that have similar pitch contours to their native lexical tones in the brainstem (Bidelman et al., 2011); and in melody discrimination and pitch memory tasks (Alexander et al., 2011; Bidelman et al., 2013). The evidence suggests that cross-domain transfer is seen following extensive experience with an acoustic cue shared by both speech and music.

Several explanations have been proposed to account for crossdomain transfer (for a review, see Asaridou and McQueen, 2013). One such possible explanation, or at least, for musicto-speech transfer, relates to the sharpening of shared auditory skills honed by extensive musical training, which may lead to musicians having a 'better ear' or generally better encoding of the input (Kraus and Chandrasekaran, 2010; Skoe and Kraus, 2012). Another possibility relates to one of the learning mechanisms

\footnotetext{
${ }^{1}$ In this paper, lexical tones are described using Chao values (Chao, 1930), where the numbers 1-5 indicate the relative height and contour of initial and final and sometimes the medial values of the tone with 1 being the lowest and 5 being the highest. For example, /ma55/ is a high level tone whereas /ma51/ is a high falling tone.
}

thought to be used in the acquisition of language and music, namely, statistical learning (Patel, 2008). Statistical learning refers to a domain-general learning mechanism in which learners acquire knowledge through extracting statistical regularities in the input. Cross-domain transfer may, result from such learning: while acquiring aspects of language or music that uses a particular acoustic cue (e.g., acquiring pitch-based elements such as lexical tones or musical pitch), learners may be more likely to show heightened attention to that cue in general. In relation to pitch, musicians and tone language listeners show an advantage in perceiving lexical tones and musical pitch, respectively, since both groups have experience in statistical learning of pitchbased elements and therefore are more likely to attend to pitch in any given input than listeners who may not have pitch experience. While it appears that non-musicians, too, use statistical learning to acquire implicit musical knowledge in their native musical system (e.g., Tillmann and McAdams, 2004; Bigand and Poulin-Charronnat, 2006; Stevens et al., 2013), non-musicians may not show cross-domain transfer relative to musicians presumably because non-musicians do not engage in statistical learning of musical pitch to the same extent as musicians. Evidence suggests that the active, purposeful nature of musical training in perceiving and producing musical pitch that musicians experience has consequences for acquiring musical knowledge more efficiently as opposed to listeners who acquire musical knowledge passively (Trainor et al., 2012).

One way to test the possibility that acquisition of speech and music plays a role in cross-domain transfer is by examining whether naïve participants show cross-domain transfer of pitch after acquiring either lexical tones or musical pitch via distributional learning, mirroring the phenomenon exhibited by tone language listeners and musicians. Distributional learning is a specific form of statistical learning that refers to acquisition of knowledge based on the distributional structure of the input encountered by the learner. Consider the case of first language acquisition: infants attend to speech sounds that they encounter in their linguistic environment and form phonological categories based on those speech sounds. For simplicity, focusing just on a continuum of voice-onset time (VOT, the duration between the release of the stop and the vibration of the vocal folds) along the range of $-120 \mathrm{~ms}$ to $+20 \mathrm{~ms}$, Hindi-learning infants will likely encounter speech sounds that can be modeled as two normal distributions with one peak at around $-85 \mathrm{~ms}$ and another at around $13 \mathrm{~ms}$ (Lisker and Abramson, 1964). English-learning infants, on the other hand, will encounter speech sounds along the same continuum that can be modeled as a single normal distribution with a peak at around $0 \mathrm{~ms}$. This difference in speech sound distributions - in particular, where the peaks occur-may explain the formation of two voicing categories by Hindi listeners (pre-voiced and voiceless) and the formation of only one voicing category by English listeners (voiceless) along that range of VOT. Distributional learning is used to account for the acquisition of the building blocks of speech and music (e.g., Maye and Gerken, 2000; Maye et al., 2002, 2008; Wanrooij et al., 2013; Escudero and Williams, 2014; Ong et al., 2015, 2016). While it remains to be seen whether distributional learning is generalizable to signals from a different domain, there are reason to believe that this 
is possible: naïve learners are able to generalize distributional learning to the same acoustic cue in a different context (Maye et al., 2008) and to different speakers (Escudero et al., 2011; Escudero and Williams, 2014) albeit within the same domain (i.e., speech). Evidence also suggests that listeners can apply a phonological principle based on one class of phonemes in their native language (e.g., short vs. long vowels) to perceive another class of phonemes (e.g., short vs. long consonants), even when the listener's native language does not use consonantal duration phonemically (Pajak and Levy, 2014). If acquiring pitchbased elements (lexical tones or musical pitch) heightens learners' attention to pitch in general, then we may expect learners to generalize such knowledge to a signal of a different domain, even if it is a different class of pitch distinction-such as a pitch contour distinction between lexical tones (mid-level tone vs. rise-fall tone) and a pitch height difference between musical pitch.

In this study, we used a distributional learning paradigm that consisted of naive adult learners' exposure to a distribution that spans either a lexical tone or a musical chord minimal pair. Within each domain, the distributions used could either facilitate the learning of two distinct sounds based on an acoustic cue that differentiates that minimal pair (a bimodal distribution, which has two modal peaks at each end of the continuum) or hinder such learning (a unimodal distribution, which has one modal peak at the center of the continuum). In previous studies, participants either listen to the training tokens passively (e.g., Escudero and Williams, 2014) or attentively (e.g., Ong et al., 2015) via a simultaneous auditory vigilance task during training. In this study, only the attentive listening training procedure is employed in order to be comparable to our previous studies on distributional learning of lexical tones and musical pitch (Ong et al., 2015, 2016). Learners' performance before and after training is assessed using an ABX discrimination task ${ }^{2}$ in both domains. In this task, participants are required to decide whether the last sound $(\mathrm{X})$ is similar to the first (A) or the second (B) on each trial. If learners engage in distributional learning, then listeners in the Bimodal condition should infer two sounds from the distribution that highlights the relevance of pitch, resulting in heightened attention to pitch after training. On the other hand, listeners in the Unimodal condition would only infer one sound from a distribution that minimizes the relevance of pitch and therefore, will not attend to pitch as much as listeners in the Bimodal condition after training. Therefore, we predict that (i) participants will show distributional learning, that is, those trained on a bimodal distribution will improve on discriminating items of the same domain as in training whereas those trained

${ }^{2}$ One may be wary of a discrimination task as a means to investigate learning of categories, instead of using both discrimination and identification tasks. In this study, where speech and music sounds are used, identification tasks using (arbitrary) labels is not ideal as precision in sound-to-label mapping may be different for speech and music, particularly since labels are not typically given to musical pitch (with the exception of absolute pitch possessors). Note that category formation does not necessarily imply 'categorical perception' of the newly formed categories, that is, a decrease in within-category and an increase in betweencategory sensitivity as a result of warping due to an abstract category. For instance, vowels are considered as separate categories even though vowels are typically perceived in a continuous manner (e.g., Fry et al., 1962). on a Unimodal distribution will not; and (ii) participants in the bimodal distribution groups will show cross-domain transfer (i.e., significant improvement on discriminating items of the other domain relative to training) whereas those in the Unimodal groups will not. Given that the $\mathrm{ABX}$ discrimination task requires listeners to use their pitch memory, pitch memory of listeners in both groups will be ascertained and controlled using a familiar song task (described below) to avoid a potential confound in the results.

\section{MATERIALS AND METHODS}

\section{Participants}

One hundred native Australian English (AusE) listeners (85 females $^{3}$; age range $=18-51, M=21.29, S D=6.04$ ) were recruited from Western Sydney University. Forty-one were monolingual AusE listeners, and 59 were bilinguals, although none spoke a tone language. Some participants $(n=20)$ reported having minimal musical training; however, in no instance was training more than 2 years ( $<1$ year, $n=1 ; 1-2$ years, $n=19$ ) and none currently practiced music. All participants reported normal hearing and no speech impairment. Participants were randomly assigned to one of four groups: Thai Unimodal, Thai Bimodal, Music Unimodal, and Music Bimodal (see Procedure of distributional learning task). These four groups are equated in terms of their age, years of musical training, proportion of female participants and monolinguals within each group (Table 1). Participants provided their written informed consent prior to participating and they were given course credit for their participation. The Western Sydney University Human Research Ethics Committee approved the study protocol.

\section{Stimuli \\ Distributional Learning Task \\ Thai stimuli}

A subset of Thai stimuli from a previous study (Ong et al., 2015) were used as speech stimuli in the distributional learning task. The target lexical tone minimal pair was Tone 33 and Tone 241. Two native Thai speakers (one female) produced the tones in a CV syllable: the female speaker produced multiple tokens of $/ \mathrm{k}^{\mathrm{h}} \mathrm{a} 33 /$ and $/ \mathrm{k}^{\mathrm{h}} \mathrm{a} 241 /$ whereas the male speaker produced multiple tokens of /na33/ and /na241/. To ensure that only the pitch contour differed between each minimal pair, we first chose a base waveform from each speaker and modified the pitch contour of other tokens from the same speaker to match the base waveform. This procedure was conducted three times for

${ }^{3}$ The larger number of female participants is representative of a larger female cohort among Psychology undergraduate students in Western Sydney University. A previous study that examined gender differences in perception of naturally produced vowels (which implies the presence of multiple acoustic cues) using a distributional learning paradigm did not reveal any gender differences in initial perception, although dividing the participants into high vs. low initial performance did reveal gender differences in integrating new cues (Wanrooij et al., 2013). Unlike the previous study, only one cue is present in each minimal pair in the present study, and therefore gender differences in integrating multiple cues is not an issue. Furthermore, there is no a priori reason to expect gender differences in pitch perception, though future research could investigate this to be sure. 
TABLE 1 | Demographic details of the four groups in this study.

\begin{tabular}{|c|c|c|c|c|c|}
\hline Demographic details & Thai unimodal & Thai bimodal & Music unimodal & Music bimodal & Statistical comparison \\
\hline Age & $21.92(4.582)$ & $22.76(8.565)$ & $20.40(6.576)$ & $20.08(2.768)$ & $F(3,96)=1.105, p=0.351$ \\
\hline $\begin{array}{l}\text { Years of Musical } \\
\text { Training }\end{array}$ & $0.480(0.7837)$ & $0.400(0.7071)$ & $0.160(0.5538)$ & $0.240(0.6633)$ & $F(3,96)=1.146, p=0.334$ \\
\hline Female & 18 & 22 & 22 & 23 & $\chi^{2}(3)=4.627, p=0.201$ \\
\hline Monolingual & 9 & 10 & 10 & 12 & $\chi^{2}(3)=0.785, p=0.853$ \\
\hline
\end{tabular}

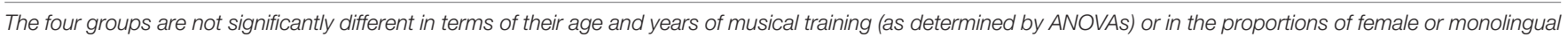
participants in each group (as determined by $x^{2}$ tests).

each Thai word, resulting in a total of 12 Thai test stimuli (2 speakers $\times 2$ Thai words $\times 3$ exemplars).

The training stimuli for this task consisted of an 8-equalstep continuum of the Male /na33/-/na241/ minimal pair. The choice of this minimal pair is motivated by our previous study (Ong et al., 2015), in which it was found this minimal pair is the most difficult to perceive compared to other Thai tone minimal pairs. We reason that the participants may benefit most from being trained on the most difficult minimal pair. The intermediate tokens were synthesized by interpolating the pitch contour on Praat (Boersma and Weenink, 2013) such that along the continuum, the pitch contour transformed from Tone 33 (Token 1) to Tone 241 (Token 8).

\section{Music stimuli}

A subset of music stimuli from a previous study (Ong et al., 2016) was used in the distributional learning task. The target musical minimal pair was a major chord transposed to a novel scale (Dean, 2009) and a 2.5\% mistuned version of that chord (e.g., Chord $\mathrm{X}-\mathrm{X}^{\prime}$ and Chord $\mathrm{Y}-\mathrm{Y}^{\prime}$ ). The frequencies of the chord were sent as MIDI via MaxMSP 5 to LogicPro 7 and realized using either a female or male choir preset ('Astral Choir' and 'Choir Male Chant,' respectively) on the Alchemy plugin. A total of four test stimuli were synthesized forming two minimal pairs: Female Chord $\mathrm{X}-\mathrm{X}^{\prime}$ and Male Chord $\mathrm{Y}-\mathrm{Y}^{\prime}$.

A continuum spanning the Female Chord $\mathrm{X}-\mathrm{X}^{\prime}$ was also synthesized by manipulating the frequency of the middle note in eight equal steps, with Token 1 being Female Chord $\mathrm{X}$ and Token 8 being Female Chord $\mathrm{X}^{\prime}$. Following from our previous study (Ong et al., 2016), we chose this minimal pair since it was reported that this minimal pair is the most difficult to perceive among the other sung minimal pairs. It is assumed that participants may benefit most from being trained on the most difficult minimal pair.

\section{Practice stimuli}

To familiarize the participants with the discrimination task, a $440 \mathrm{~Hz}$ sinewave tone and a $440 \mathrm{~Hz}$ sawtooth tone, both $800 \mathrm{~ms}$, were synthesized using Praat (Boersma and Weenink, 2013). In addition, the sinewave tone was used as 'beeps' during training as part of a concurrent auditory vigilance task (Ong et al., 2015).

\section{Familiar Song Task}

A familiar song task was used to measure participants' pitch memory. Forty popular English songs as determined from a pilot study were chosen and the first $5 \mathrm{~s}$ of each song (i.e., the instrumental portion) was excised and duplicated. Half the duplications had their pitch raised whereas the other half had their pitch lowered, each with a transposition of either one or two semitones, resulting in four sets of stimuli $(+1,+2,-1$, -2 ) with each set having 10 songs. For the original excerpts, we manipulated the pitch upward and then downward to the same degree as their duplications in order to remove any artifacts of digital manipulation (Schellenberg and Trehub, 2003).

\section{Equipment}

All the tasks were presented using MATLAB 2012b on an Acer TravelMate P653 laptop. The auditory stimuli were presented using a pair of Sennheiser HD650 headphones connected to an Edirol USB Audio Capture UA-25EX audio interface.

\section{Procedure}

Participants completed three tasks (distributional learning task, familiar song task, and language and music background questionnaire), the order of which was randomized across participants. The entire experiment took approximately $50 \mathrm{~min}$ to complete.

\section{Distributional Learning Task}

There were three phases to the distributional learning task: pretest, training, and posttest. At pretest and at posttest, participants completed an ABX discrimination task (e.g., in a trial in which participants hear/na33/-/na241/-/na33/, the correct answer is $\mathrm{A}$, or the first sound) for test stimuli of both domains. There were 32 trials per test phase, with eight repetitions of the four target minimal pairs ( 2 Thai lexical tones +2 musical chord minimal pairs). The test trials were not blocked by test domain but presented in a randomized order for each participant. Participants were required to respond within $1 \mathrm{~s}$ in order to maintain vigilance and there were no replacement trials for slow responses. Prior to pretest, participants were given four practice trials with feedback.

During training, participants were randomly assigned to be trained on either Thai lexical tones or novel musical chords and then further assigned to either a Unimodal or a Bimodal condition within each domain. Listeners in both distribution conditions heard the same number of training tokens (i.e., 256) but the conditions featured different modal peaks of the distribution; listeners in the Unimodal condition heard Tokens 4 and 5 most frequently whereas those in the Bimodal condition heard Tokens 2 and 7 most frequently (Figure 1). Listeners in both conditions were presented with Tokens 1 and 8 (i.e., the stimuli used in pretest and posttest for the minimal pairs for 


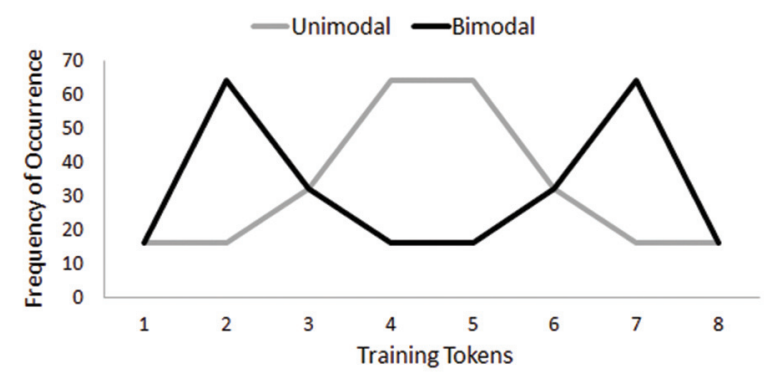

FIGURE 1 | Frequency of occurrence for each training token heard by the Unimodal condition and the Bimodal condition.

both domains) the same number of times. In order to ensure that participants listened to the entire set of training tokens, they were required to complete a cover task-to mark on a response sheet with the numbers 1-288 whenever they heard a 'beep' by circling the sound number to which the 'beep' corresponded (Ong et al., 2015). A total of 32 beeps were interspersed randomly within the training tokens. The training phase took approximately $6 \mathrm{~min}$ to complete.

\section{Familiar Song Task}

In each trial of the familiar song task, participants were shown a song title and the artist who performed the song and were asked to indicate whether they were familiar with the song. If they were unfamiliar with the song, they moved on to the next trial with no replacement trials. If they were familiar with the song, they were then presented with two excerpts: an original and a transposed version, the order of which was randomized, and they were required to choose which excerpt was the original. There were two blocks of 20 trials, with one block corresponding to a pitch transposition of one semitone and the other of two semitones. The presentation order between the blocks and the trials within each block was randomized. Across all participants, the average number of songs chosen as familiar, and hence, presented to the participants, was $27.96(S D=6.61$, Range $=10-40)$.

\section{Language and Musical Background Questionnaire}

In the language and musical background questionnaire, participants were asked to provide their demographic details as well as to list all the languages known to them and rate on a 5-point scale how well they (i) read, (ii) speak, (iii) write, and (iv) understand each of those languages. They were also asked to indicate whether they have had musical training and if so, the age of commencement, the duration of training in years, and the instruments played.

\section{RESULTS}

All participants correctly identified with $100 \%$ accuracy the 'beeps' in the concurrent auditory vigilance task during training, so no participant's data were excluded from analysis. Prior to analyzing the results of the distributional learning task, we first examined participants' performance (i) on the familiar song task, and (ii) during Pretest. Concerning the familiar song task, performance ranged from 0 to $94.44 \%(M=64.59 \%$, $S D=14.34 \%$ ), with eight participants (Unimodal $=5$, Bimodal = 3) meeting the Absolute Pitch criterion (i.e., accuracy of at least 85\%; Deutsch et al., 2004). Importantly, the two distribution conditions did not differ overall in their pitch memory performance $[t(98)=1.493, p=0.139]$. Comparison of the two distribution conditions' Pretest scores revealed no significant difference $[t(98)=0.223, p=0.824]$. Thus, both distribution conditions are similar in their pitch memory and in their perception of lexical tones and musical pitch; therefore, any differences between the two conditions at Posttest can be attributed to the training itself.

To deal with potential differences between Thai and music stimuli, we determined whether the stimuli from both domains are similar in difficulty by first conducting a mixed ANOVA with between-subject factors Distribution Condition (Unimodal vs. Bimodal) and Training Domain (Thai vs. Music) and within-subject factors Session (Pretest vs. Posttest) and Test items (Thai vs. Music). There was a main effect of Session $\left[F(1,96)=42.196, p<0.001, \eta_{\mathrm{p}}^{2}=0.305\right]$ : Posttest scores $(M=0.621, S E=0.012)$ were higher than Pretest scores $(M=0.548, S E=0.011)$. There was also an interaction between Test items and Training Domain $[F(1,96)=71.819, p<0.001$, $\left.\eta_{\mathrm{p}}^{2}=0.428\right]$ : for those who were trained on Thai stimuli, same domain test items (Thai tones, $M=0.653, S E=0.016$ ) were easier to discriminate than cross-domain test items (sung chords, $M=0.524, S E=0.018$ ) whereas for those trained on Music stimuli, cross-domain test items (Thai tones, $M=0.639$, $S E=0.019)$ were easier to discriminate than same domain test items (Thai tones, $M=0.521, S E=0.016$ ). Since Thai tones were easier to discriminate than musical chords in general, two separate sets of analyses-one for each training domain-were conducted, as participants' (in)ability to perceive the stimuli, either during training or test, may affect distributional learning (Frost et al., 2015). For each set of analyses, we conducted a mixed ANOVA (to determine whether there was a Session * Distribution Condition interaction) and a series of one-sample $t$-tests. The former examines whether the distribution groups significantly differed in general in their Pretest vs. Posttest scores (collapsing across the different test domains); whereas the latter provides information on whether there was any improvement, as indicated by whether posttest minus pretest scores (i.e., difference scores) differed from zero in each particular test domain ${ }^{4}$.

\section{Thai Tone Training Group}

Pretest scores in the Thai tone training group for the two distribution conditions did not differ significantly $[t(48)=0.857$, $p=0.396]$, indicating that any difference at Posttest would be

${ }^{4}$ It should also be noted that given the current experimental design (pretesttraining-posttest), some improvement is to be expected due to practice, irrespective of the distribution encountered during training (Ong et al., 2015). However, if the Unimodal group fails to show significant improvement despite the pretest-training-posttest design, then this lack of improvement due to practice can be taken as a stronger evidence of distributional learning over and above the improvement that is predicted for the Bimodal condition. 


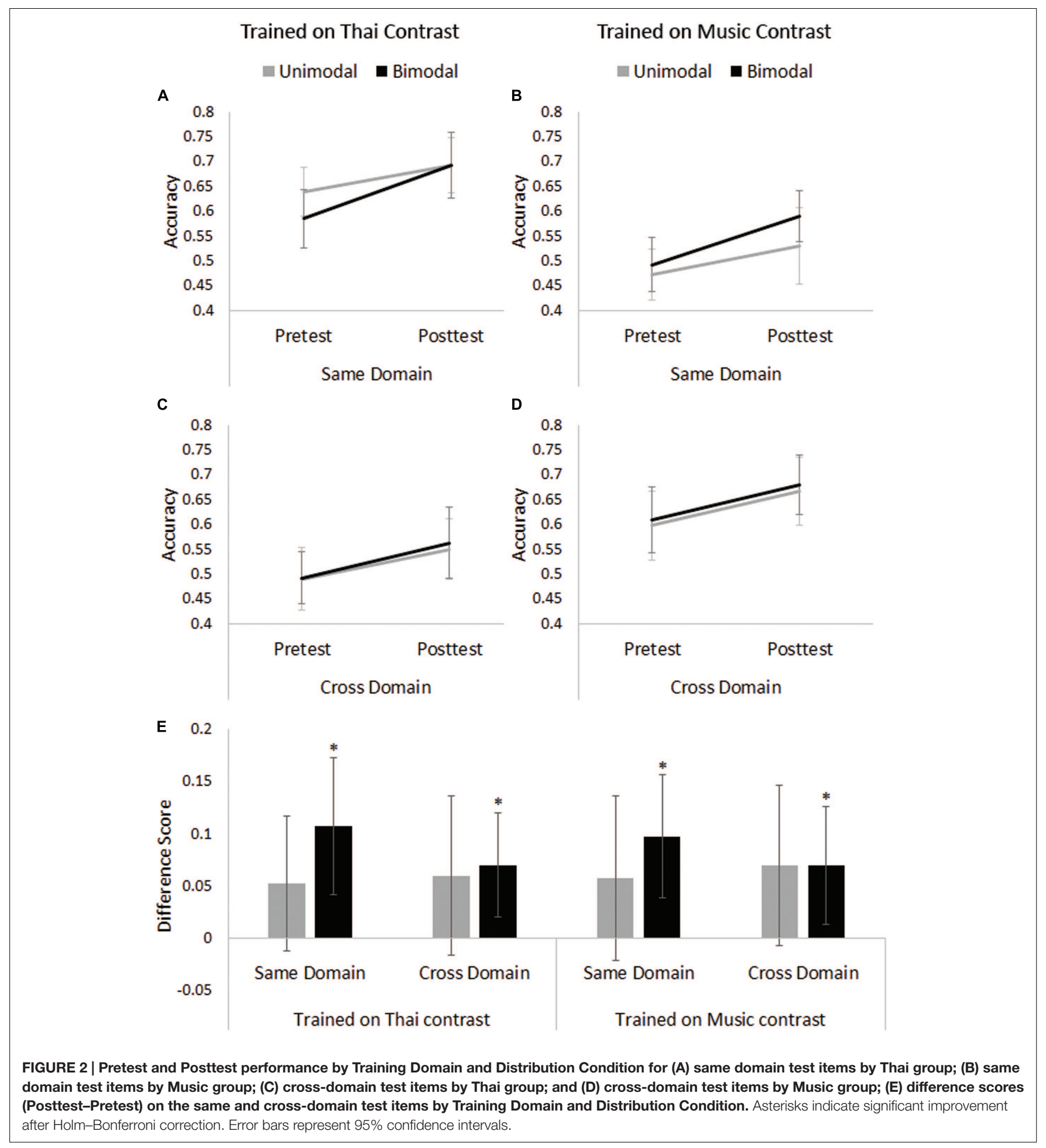

due to differences in training. Figures 2A,C illustrate Pretest and Posttest performance on same and cross-domain test items, respectively. The ANOVA revealed a main effect of Session $\left[\left(F(1,48)=21.993, p<0.001, \eta_{\mathrm{p}}^{2}=0.314\right]\right.$ : in general Posttest scores $(M=0.624, S E=0.016)$ were higher than Pretest Scores $(M=0.552, S E=0.015)$. There was also a main effect of Test
Domain $\left[F(1,48)=37.665, p<0.001, \eta_{p}^{2}=0.440\right]$, showing that performance was higher on Thai stimuli (Same Domain; $M=0.653, S E=0.016$ ) than music stimuli (Cross Domain; $M=0.524, S E=0.018)$. However, there was no significant Session $*$ Distribution Condition interaction $[F(1,48)=1.105$, $\left.p=0.298, \eta_{\mathrm{p}}^{2}=0.023\right]$. 
Following previous distributional learning studies (e.g., Escudero et al., 2011; Ong et al., 2015), we conducted four onesample $t$-tests with Holm-Bonferroni correction on difference scores (i.e., Posttest-Pretest scores) to determine whether the improvement from Pretest to Posttest on each of the Test Domains was significantly different from zero (where zero means no improvement) for both distribution conditions. For the Bimodal Thai group, there was improvement from Pretest to Posttest on discriminating test items within the same domain [i.e., Thai stimuli, $t(24)=3.384, p=0.002$, adjusted $\alpha=0.025$, Cohen's $d=0.68$ ] and across to the other domain [i.e., music stimuli, $t(24)=2.914, p=0.008$, adjusted $\alpha=0.05$ Cohen's $d=0.58$; see Figure 2E]. Conversely, the Unimodal Thai group did not improve on test items for either domain [Thai stimuli, $t(24)=1.672, p=0.107$, adjusted $\alpha=0.025$, Cohen's $d=0.33$; music stimuli, $t(24)=1.619, p=0.119$, adjusted $\alpha=0.05$, Cohen's $d=0.32$.

\section{Musical Chord Training Group}

Pretest scores in the musical chord training group for the two distribution conditions did not differ significantly $[t(48)=0.493$, $p=0.624]$, indicating that any difference at Posttest would be due to differences in training. Figures 2B,D illustrate Pretest and Posttest performance on same and cross-domain test items, respectively. The ANOVA revealed significant main effects of Session $\left[F(1,48)=20.302, p<0.001, \eta_{\mathrm{p}}^{2}=0.297\right]$ and Test Domain $\left[F(1,48)=34.154, p<0.001, \eta_{p}^{2}=0.416\right]$, mirroring the results of those trained on Thai tones. In general, Posttest scores $(M=0.617, S E=0.018)$ were higher than Pretest scores $(M=0.543, S E=0.016)$ and performance on Thai stimuli (Cross Domain, $M=0.639, S E=0.019)$ were higher than music stimuli (Same Domain, $M=0.521, S E=0.016)^{5}$. In addition, just as for Thai tones, there was no significant Session ${ }^{*}$ Distribution Condition interaction $\left[F(1,48)=0.373, p=0.544, \eta_{\mathrm{p}}^{2}=0.008\right]$.

One-sample $t$-tests indicated that, after Holm-Bonferroni correction, the Bimodal Music group improved on test stimuli of the same domain [i.e., music stimuli, $t(24)=3.429, p=0.002$, adjusted $\alpha=0.025$, Cohen's $d=0.69$ ] and on test stimuli of the other domain [i.e., Thai stimuli, $t(24)=2.562, p=0.017$, adjusted $\alpha=0.05$, Cohen's $d=0.51]$ whereas the Unimodal Music group did not show improvement following training on either of the Test Domains [music stimuli, $t(24)=1.506, p=0.145$, adjusted $\alpha=0.05$, Cohen's $d=0.30$; Thai stimuli, $t(24)=1.881, p=0.072$, adjusted $\alpha=0.025$, Cohen's $d=0.38$; see Figure 2E].

\section{DISCUSSION}

The results of this study provide evidence for distributional learning of pitch-based distinctions (lexical tones and

\footnotetext{
${ }^{5}$ The somewhat counterintuitive finding that performance on the cross-domain test items is higher than the same domain test items for the music training group reflects the fact that our Thai stimuli were easier to perceive than the music stimuli, a finding that was found consistently in all our analyses in this paper. One possibility for such finding may relate to the unfamiliar nature of Thai lexical tones relative to sung chords to non-tone language listeners, who may not be subjected to interference for the Thai tones due to their unfamiliarity.
}

musical pitch): bimodal distribution training conditions led to improvement in discriminating minimal pairs of the same domain whereas Unimodal conditions showed suppression of any improvement due to training. This twofold outcome is consistent with our previous studies on distributional learning of lexical tones and musical pitch (Ong et al., 2015, 2016). However, we did not find significant interactions between Session and Distribution Condition in the mixed ANOVA, contrary to our previous studies (Ong et al., 2015, 2016) that reported a general (collapsing across test items) pre-post improvement for the Bimodal condition but not the Unimodal condition. This may be due to a number of reasons including individual differences in perceiving pitch, which could result in a large variance in performance (Demany and Semal, 2002; de Cheveigné, 2012). Furthermore, this study is different from previous studies in its test stimulus set variability. Similar to previous studies, we had 32 trials in each test phase. However, half the trials in this study were on two Thai tone minimal pairs (Male /na33/-/na241/ and Female $\left./ \mathrm{k}^{\mathrm{h}} \mathrm{a} 33 /-/ \mathrm{k}^{\mathrm{h}} \mathrm{a} 241 /\right)$ and the other half on two musical chord minimal pairs (Female Chord $\mathrm{X}-\mathrm{X}^{\prime}$ and Male Chord $\mathrm{Y}-\mathrm{Y}^{\prime}$ ). In previous studies, all 32 trials were on four different minimal pairs of the same domain. In other words, there was less variability in the test stimuli within the same domain in this study compared to those in our previous studies, which may have weakened the effect of distributional learning. Indeed, it has been suggested that stimulus variability facilitates learning of Mandarin lexical tones, at least among high-aptitude learners (Sadakata and McQueen, 2014) and so this may account for a weaker distributional learning effect in this study relative to previous studies on distributional learning of pitch.

With regard to cross-domain transfer, the results revealed that participants who were trained on a bimodal distribution, but not those trained on a unimodal distribution, showed improvement in discriminating test items of the other domain. We propose that the observed cross-domain transfer among participants in the Bimodal conditions is due to the reliability of the pitch cue in separating the two sounds/end points in the distribution, leading to heightened attention to pitch in general. Conversely, participants in the Unimodal conditions failed to exhibit any cross-domain transfer presumably because pitch was minimized in a distribution from which only one sound was inferred and therefore those learners did not show heightened attention to pitch. The cross-domain transfer in this study was found despite qualitative pitch differences between the two domains; the lexical tone minimal pair is a difference in pitch contour whereas the musical chord minimal pair is a difference in pitch height of the middle note. Our results suggest that distributional learning of pitch-based elements (lexical tones or musical pitch) may explain the cross-domain transfer seen among 'pitch experts' (e.g., Pfordresher and Brown, 2009; Herrera et al., 2011; Bidelman et al., 2013; Burnham et al., 2014).

The cross-domain transfer found in this study is reminiscent of findings in speech perception research in which listeners are able to transfer a learned skill at using an acoustic cue phonemically in their native language to discriminate a nonnative contrast, even if the non-native contrast is of a separate 
class of phonemes (Pajak and Levy, 2014). Moreover, previous distributional learning studies have demonstrated that after being exposed to a bimodal distribution spanning a consonant contrast differentiated by VOT, infant learners (Maye et al., 2008), but not adult learners (Maye and Gerken, 2001), could generalize their knowledge to a VOT contrast on a different place of articulation (e.g., improved discrimination of $/ \mathrm{g} /-/ \mathrm{k} /$ following bimodal training on $/ \mathrm{d} /-/ \mathrm{t} /$ ) whereas those trained on a Unimodal distribution were not able to do so. In the present study, adult learners did show generalization to a different domain, at least when a common acoustic cue is used across both domains. The difference in results between the present study and Maye and Gerken (2001) may be due to methodological differences (e.g., the use of attentive distributional learning in this study as opposed to passive distributional learning in Maye and Gerken, 2001) and/or differences in the target acoustic cue. Nonetheless, our results suggest that distributional learning is generalizable to a different domain.

While we have found some evidence of transfer between speech and music, these results raise the question of the extent to which the signal needs to be similar in order for transfer to occur. One possibility is that the learner cognitively compares the acoustic signals and if the relevant features are deemed similar enough, then the same processing will be applied to both signals. This would be in line with the proposal that speech and music, despite having very different representations and output, share similar processing mechanisms since both domains share similar acoustic cues (Patel, 2008). The proposal is complemented by the fact that the auditory system appears to be agnostic with respect to the nature of the signal in its early stage of processing (i.e., in the brainstem; Krishnan et al., 2010; Bidelman et al., 2011) and presumably not until much later during processing is the signal's cognitive function defined. Therefore, it could be argued that crossdomain transfer may be dependent on whether the features of interest that define the signals are deemed similar enough by the learner.

On the other hand, it is possible that participants in this study did not treat the stimuli (lexical tones and sung musical chords) as belonging to different domains, and therefore did not actually show any cross-domain transfer at all. While, acoustically, there appear to be some differences between speech and song-for example, articulation is often more precise in speech than in song and vowel duration tends to be longer in song than in speech (Seidner and Wendler, 1978, as cited in Merrill et al., 2012) these characteristics may not be as evident if the signal is short in duration, such as in the case of isolated lexical tones or a sung note or chord. Moreover, participants' functional interpretation of the signal may change under certain conditions-for example, a speech signal may suddenly be interpreted as someone singing when presented repeatedly (Deutsch et al., 2011). Thus, lexical tones, when presented repeatedly and in an isolated context, could be interpreted as someone singing and perceived as music-like. Indeed, tone language listeners but not non-tone language listeners show left lateralization of processing lexical tones whereas both groups show right lateralization of processing hummed version of lexical tones (Van Lancker and Fromkin, 1973, 1978; Van Lancker, 1980). This suggests that tone-language listeners treat isolated lexical tones to be speech-like more than do non-tone language listeners, who may treat isolated lexical tones to be more music-like. In contrast, Burnham et al. (2014) found that non-tone language non-musicians seem to treat lexical tones to be speech-like, at least when lexical tones are presented with two other sets of stimuli: low-pass filtered lexical tones (i.e., tones that do not convey any phonological elements of speech and consist only of frequencies below $270 \mathrm{~Hz}$ ) and violin notes that approximate the pitch contour of the lexical tones. In other words, it is possible that the participants in Burnham et al. (2014) may have treated lexical tones to be speech-like relative to the other stimuli that are presumably interpreted as more music-like. Thus, it remains plausible that the participants in the present experiment may consider isolated lexical tones and synthesized sung chords to be from the same domain.

To eliminate the possibility that participants in the present study perceived lexical tones and sung chords to be from the same domain, we conducted a stimulus identification pilot study with a different set of participants from the same population (i.e., AusE non-musicians, $n=23$ ). Participants were required to decide whether the signal was 'spoken' or 'sung' and then rate their confidence on a 5 -point scale $(1=$ "somewhat confident" and $5=$ "very confident") without any feedback. On every trial, we scored the participants based on how confident they were: participants were given a positive score that corresponds to their confidence rating if they were correct and a negative score that corresponds to their confidence rating if they were incorrect. Two overall scores were obtained for each participant, one corresponding to each domain. The results revealed that participants perceived isolated Thai lexical tones as 'speech' and synthesized sung chords as 'sung' at rates above chance [Thai lexical tones: $t(22)=5.052, p<0.001$; sung chords: $t(22)=20.607, p<0.001]$. However, performance on lexical tones $(M=1.93, S E=0.38)$ was significantly lower than on sung chords $[M=4.07, S E=0.20 ; t(22)=5.745, p<0.001]$, suggesting that as we suspected, isolated lexical tones are more ambiguous for non-tone language non-musicians than are sung chords. Taken together, this suggests that despite being less confident and less accurate in identifying lexical tones as spoken, the participants treated the stimuli as intended, which provides us with some assurance that the participants in the main experiment treated the isolated lexical tones as speech and therefore indeed demonstrated cross-domain transfer ${ }^{6}$.

${ }^{6}$ It is possible that our choice of sung chords as a musical medium may be considered speech-like and hence bias the results toward cross-domain transfer. While that is certainly a possibility, the sung chords used in this study consist only of vowels which do not have any lexical meaning. Furthermore, as revealed in the stimuli identification task, the sung chords are indeed perceived to be sung and distinct from speech. Therefore, the sung chords are perceived to be musical by our participants and presumably have the same status as musical tones produced from musical instruments. The experiment could certainly be repeated with tones from musical instruments and it is certainly worth investigating in the future whether there might be differences in cross-domain transfer if the musical stimuli are sung or musical instruments. 


\section{CONCLUSION}

Our results indicate that naïve learners show distributional learning of lexical tones and musical pitch, thus replicating our previous results (Ong et al., 2015, 2016). More importantly, we demonstrated that distributional learning may play a role in cross-domain transfer: in this study, cross-domain transfer was only observed among learners who inferred a pitch distinction in the input (such as learners in the bimodal condition), which presumably led those learners to show heightened attention to pitch in general. These results are indicative of cross-domain transfer, especially given that a follow-up study provides evidence that the lexical tone and musical chord stimuli were identified accurately according to their specific domain. The effect of distributional learning appears to be smaller in this study than that in our previous studies. This may be due to large individual differences in pitch perception and the lack of variability in the test stimuli within each domain. Nonetheless, our findings suggest that acquiring speech or musical items based on subtle pitch differences may lead to cross-domain transfer of pitch.

\section{REFERENCES}

Alexander, J. A., Bradlow, A. R., Ashley, R. D., and Wong, P. C. M. (2011). "Music-melody perception in tone-language and non-tone-language speakers," in Proceedings of the 156th Meeting of the Acoustical Society of America, Miami, FL, 1-4.

Alexander, J. A., Wong, P. C. M., and Bradlow, A. R. (2005). "Lexical tone perception in musicians and non-musicians," in Proceedings of the Interspeech 2005 (Lisbon: ISCA Archive), 397-400.

Asaridou, S. S., and McQueen, J. M. (2013). Speech and music shape the listening brain: evidence for shared domain-general mechanisms. Front. Psychol. 4:321. doi: 10.3389/fpsyg.2013.00321

Besson, M., Chobert, J., and Marie, C. (2011). Transfer of training between music and speech: common processing, attention, and memory. Front. Psychol. 2:94. doi: $10.3389 /$ fpsyg.2011.00094

Bidelman, G. M., and Alain, C. (2015). Musical training orchestrates coordinated neuroplasticity in auditory brainstem and cortex to counteract age-related declines in categorical vowel perception. J. Neurosci. 35, 1240-1249. doi: 10.1523/JNEUROSCI.3292-14.2015

Bidelman, G. M., Gandour, J. T., and Krishnan, A. (2011). Musicians and tone-language speakers share enhanced brainstem encoding but not perceptual benefits for musical pitch. Brain Cogn. 77, 1-10. doi: 10.1016/j.bandc.2011.07.006

Bidelman, G. M., Hutka, S., and Moreno, S. (2013). Tone language speakers and musicians share enhanced perceptual and cognitive abilities for musical pitch: evidence for bidirectionality between the domains of language and music. PLoS ONE 8:e60676. doi: 10.1371/journal.pone.0060676

Bigand, E., and Poulin-Charronnat, B. (2006). Are we "experienced listeners"? A review of the musical capacities that do not depend on formal musical training. Cognition 100, 100-130. doi: 10.1016/j.cognition.2005. 11.007

Boersma, P., and Weenink, D. (2013). Praat: Doing Phonetics by Computer. Available at: http://www.praat.org

Burnham, D., Brooker, R., and Reid, A. (2014). The effects of absolute pitch ability and musical training on lexical tone perception. Psychol. Music 43, 1-17. doi: $10.1177 / 0305735614546359$

Chao, Y.-R. (1930). A system of tone-letters. Maître Phon. 45, 24-27.

Chobert, J., Francois, C., Velay, J. L., and Besson, M. (2014). Twelve months of active musical training in 8-to 10-year-old children enhances the preattentive processing of syllabic duration and voice onset time. Cereb. Cortex 24, 956-967. doi: $10.1093 /$ cercor/bhs377

\section{AUTHOR CONTRIBUTIONS}

JO posed the research question and designed the experiment with useful feedback from DB, PE, and CS. JO prepared the stimuli, programmed the experiment, recruited the participants, conducted the experiment, and performed the data analyses. JO prepared the manuscript with valuable input from DB, CS, and PE.

\section{FUNDING}

JO was supported by The MARCS Institute Research Training Scheme.

\section{ACKNOWLEDGMENT}

The authors are grateful to Roger Dean for his advice on preparing the musical stimuli.

de Cheveigné, A. (2012). "Pitch perception," in The Oxford Handbook of Auditory Science: Hearing, ed. C. J. Plack (Oxford: Oxford University Press), 71-104. doi: 10.1093/oxfordhb/9780199233557.013.0004

Dean, R. T. (2009). Widening unequal tempered microtonal pitch space for metaphoric and cognitive purposes with new prime number scales. Leonardo 42, 94-95. doi: 10.1162/leon.2009.42.1.94

Demany, L., and Semal, C. (2002). Learning to perceive pitch differences. J. Acoust. Soc. Am. 111, 1377-1388. doi: 10.1121/1.1445791

Deutsch, D., Henthorn, T., and Dolson, M. (2004). Absolute pitch, speech, and tone language: some experiments and a proposed framework. Music Percept. 21, 339-356. doi: 10.1525/mp.2004.21.3.339

Deutsch, D., Henthorn, T., and Lapidis, R. (2011). Illusory transformation from speech to song. J. Acoust. Soc. Am. 129, 2245-2252. doi: 10.1121/1.3562174

Elmer, S., Meyer, M., and Jäncke, L. (2012). Neurofunctional and behavioral correlates of phonetic and temporal categorization in musically trained and untrained subjects. Cereb. Cortex 22, 650-658. doi: 10.1093/cercor/ bhr 142

Escudero, P., Benders, T., and Wanrooij, K. (2011). Enhanced bimodal distributions facilitate the learning of second language vowels. J. Acoust. Soc. Am. 130, EL206-EL212. doi: 10.1121/1.3629144

Escudero, P., and Williams, D. (2014). Distributional learning has immediate and long-lasting effects. Cognition 133, 408-413. doi: 10.1016/j.cognition. 2014.07.002

François, C., Chobert, J., Besson, M., and Schön, D. (2012). Music training for the development of speech segmentation. Cereb. Cortex 23, 2038-2043. doi: $10.1093 /$ cercor/bhs 180

Frost, R., Armstrong, B. C., Siegelman, N., and Christiansen, M. H. (2015). Domain generality versus modality specificity: the paradox of statistical learning. Trends Cogn. Sci. 19, 117-125. doi: 10.1016/j.tics.2014.12.010

Fry, D. B., Abramson, A. S., Eimas, P. D., and Liberman, A. M. (1962). The identification and discrimination of synthetic vowels. Lang. Speech 5, 171-189.

Herrera, L., Lorenzo, O., Defior, S., Fernandez-Smith, G., and Costa-Giomi, E. (2011). Effects of phonological and musical training on the reading readiness of native- and foreign-Spanish-speaking children. Psychol. Music 39, 68-81. doi: 10.1177/0305735610361995

Kraus, N., and Chandrasekaran, B. (2010). Music training for the development of auditory skills. Nat. Rev. Neurosci. 11, 599-605. doi: 10.1038/ nrn2882

Krishnan, A., Gandour, J. T., Smalt, C. J., and Bidelman, G. M. (2010). Languagedependent pitch encoding advantage in the brainstem is not limited to 
acceleration rates that occur in natural speech. Brain Lang. 114, 193-198. doi: 10.1016/j.bandl.2010.05.004

Kühnis, J., Elmer, S., Meyer, M., and Jäncke, L. (2013). The encoding of vowels and temporal speech cues in the auditory cortex of professional musicians: an EEG study. Neuropsychologia 51, 1608-1618. doi: 10.1016/j.neuropsychologia.2013.04.007

Lisker, L., and Abramson, A. S. (1964). A cross-language study of voicing in initial stops: acoustical measurements. Word 20, 384-422. doi: 10.1080/00437956. 1964.11659830

Marie, C., Delogu, F., Lampis, G., Belardinelli, M. O., and Besson, M. (2011). Influence of musical expertise on segmental and tonal processing in Mandarin Chinese. J. Cogn. Neurosci. 23, 2701-2715. doi: 10.1162/jocn.2010. 21585

Marie, C., Kujala, T., and Besson, M. (2012). Musical and linguistic expertise influence pre-attentive and attentive processing of non-speech sounds. Cortex 48, 447-457. doi: 10.1016/j.cortex.2010.11.006

Maye, J., and Gerken, L. (2000). "Learning phonemes without minimal pairs," in Proceedings of the 24th Annual Boston University Conference on Language Development (Princeton, NJ: Citeseer), 522-533.

Maye, J., and Gerken, L. (2001). "Learning phonemes: how far can the input take us," in Proceedings of the 25th Annual Boston University Conference on Language Development (Somerville, MA: Cascadilla Press), 480-490.

Maye, J., Weiss, D. J., and Aslin, R. N. (2008). Statistical phonetic learning in infants: facilitation and feature generalization. Dev. Sci. 11, 122-134. doi: 10.1111/j.1467-7687.2007.00653.x

Maye, J., Werker, J. F., and Gerken, L. (2002). Infant sensitivity to distributional information can affect phonetic discrimination. Cognition 82, B101-B111. doi: 10.1016/S0010-0277(01)00157-3

Merrill, J., Sammler, D., Bangert, M., Goldhahn, D., Lohmann, G., Turner, R., et al. (2012). Perception of words and pitch patterns in song and speech. Front. Psychol. 3:76. doi: 10.3389/fpsyg.2012.00076

Musacchia, G., Sams, M., Skoe, E., and Kraus, N. (2007). Musicians have enhanced subcortical auditory and audiovisual processing of speech and music. Proc. Natl. Acad. Sci. U.S.A. 104, 15894-15898. doi: 10.1073/pnas.07014 98104

Ong, J. H., Burnham, D., and Escudero, P. (2015). Distributional learning of lexical tones: a comparison of attended vs. unattended listening. PLoS ONE 10:e133446. doi: 10.1371/journal.pone.0133446

Ong, J. H., Burnham, D., and Stevens, C. J. (2016). Learning novel musical pitch via distributional learning. J. Exp. Psychol. Learn. Mem. Cogn. doi: 10.1037/xlm0000286 [Epub ahead of print].

Ott, C. G. M., Langer, N., Oechslin, M. S., Meyer, M., and Jäncke, L. (2011). Processing of voiced and unvoiced acoustic stimuli in musicians. Front. Psychol. 2:195. doi: 10.3389/fpsyg.2011.00195

Pajak, B., and Levy, R. (2014). The role of abstraction in non-native speech perception. J. Phon. 46, 147-160. doi: 10.1016/j.wocn.2014.07.001

Patel, A. D. (2003). Language, music, syntax and the brain. Nat. Neurosci. 6, 674-681. doi: 10.1038/nn1082

Patel, A. D. (2008). Music, Language, and the Brain. Oxford: Oxford University Press.

Pfordresher, P. Q., and Brown, S. (2009). Enhanced production and perception of musical pitch in tone language speakers. Atten. Percept. Psychophys. 71, 1385-1398. doi: 10.3758/APP.71.6.1385
Sadakata, M., and McQueen, J. M. (2014). Individual aptitude in Mandarin lexical tone perception predicts effectiveness of high-variability training. Front. Psychol. 5:1318. doi: 10.3389/fpsyg.2014.01318

Schellenberg, E. G., and Trehub, S. E. (2003). Good pitch memory is widespread. Psychol. Sci. 14, 262-266. doi: 10.1111/1467-9280.03432

Seidner, W., and Wendler, J. (1978). Die Sängerstimme. Berlin: Henschel Verlag.

Skoe, E., and Kraus, N. (2012). "Human subcortical auditory function provides a new conceptual framework for considering modularity," in Language and Music as Cognitive Systems, eds P. Rebuschat, M. Rohrmeier, J. A. Hawkins, and I. Cross (Oxford: Oxford Scholarship), 301-315.

Slevc, L. R., and Miyake, A. (2006). Individual differences in second-language proficiency: does musical ability matter? Psychol. Sci. 17, 675-681. doi: 10.1111/j.1467-9280.2006.01765.x

Stevens, C. J., Tardieu, J., Dunbar-Hall, P., Best, C. T., and Tillmann, B. (2013). Expectations in culturally unfamiliar music: influences of proximal and distal cues and timbral characteristics. Front. Psychol. 4:789. doi: 10.3389/fpsyg.2013.00789

Tillmann, B., and McAdams, S. (2004). Implicit learning of musical timbre sequences: statistical regularities confronted with acoustical (dis)similarities. J. Exp. Psychol. Learn. Mem. Cogn. 30, 1131-1142. doi: 10.1037/02787393.30.5.1131

Trainor, L. J., Marie, C., Gerry, D., Whiskin, E., and Unrau, A. (2012). Becoming musically enculturated: effects of music classes for infants on brain and behavior. Ann. N. Y. Acad. Sci. 1252, 129-138. doi: 10.1111/j.17496632.2012.06462.x

Van Lancker, D. (1980). Cerebral lateralization of pitch cues in the linguistic signal. Pap. Linguist. 13, 201-249. doi: 10.1080/08351818009370498

Van Lancker, D., and Fromkin, V. A. (1973). Hemispheric specialization for pitch and "tone": evidence from Thai. J. Phon. 1, 101-109.

Van Lancker, D., and Fromkin, V. A. (1978). Cerebral dominance for pitch contrasts in tone language speakers and in musically untrained and trained English speakers. J. Phon. 6, 19-23.

Wanrooij, K., Escudero, P., and Raijmakers, M. E. J. (2013). What do listeners learn from exposure to a vowel distribution? An analysis of listening strategies in distributional learning. J. Phon. 41, 307-319. doi: 10.1016/j.wocn.2013.03.005

Wong, P. C. M., and Perrachione, T. K. (2007). Learning pitch patterns in lexical identification by native English-speaking adults. Appl. Psychol. 28, 565-585. doi: $10.1017 / \mathrm{s} 0142716407070312$

Wong, P. C. M., Skoe, E., Russo, N. M., Dees, T. M., and Kraus, N. (2007). Musical experience shapes human brainstem encoding of linguistic pitch patterns. Nat. Neurosci. 10, 420-422. doi: 10.1038/nn1872

Yip, M. (2002). Tone. Cambridge: Cambridge University Press. doi: 10.1017/ CBO9781139164559

Conflict of Interest Statement: The authors declare that the research was conducted in the absence of any commercial or financial relationships that could be construed as a potential conflict of interest.

Copyright (c) 2016 Ong, Burnham, Stevens and Escudero. This is an open-access article distributed under the terms of the Creative Commons Attribution License (CC BY). The use, distribution or reproduction in other forums is permitted, provided the original author(s) or licensor are credited and that the original publication in this journal is cited, in accordance with accepted academic practice. No use, distribution or reproduction is permitted which does not comply with these terms. 